\title{
Knowledge Attitude and Practices Regarding Personal Hygiene among the Male Primary School Children in Abha, Kingdom of Saudi Arabia: A Cross-Sectional Study
}

\author{
${ }^{* 1}$ Ahmed Hazazi, ${ }^{2}$ Sriram Chandramohan, ${ }^{3}$ Javed Khan, ${ }^{4}$ Mohammed AL-Mohaithef \\ 1,2,3 Lecturer, Department of Public Health, College of Health Sciences, Saudi Electronic University, Abha \\ Branch, Kingdom of Saudi Arabia, ${ }^{4}$ Assistant Professor, Department of Public Health, College of Health \\ Sciences, Saudi Electronic University, Dammam Branch, Kingdom of Saudi Arabia. \\ *Email: a.Hazazi@seu.edu.sa
}

Received: 04 ${ }^{\text {th }}$ December 2017, Accepted: $14^{\text {th }}$ December 2017, Published: 28th February 2018

\begin{abstract}
Background: Lack of knowledge about personal hygiene and poor hygienic practices increases the burden of communicable diseases. Maintaining a good personal hygiene among children helps to improve the quality of life and also longevity. Therefore the objective of the current study is to assess the knowledge attitude and practices of personal hygiene and also to evaluate the relationship between knowledge attitude and practices. Methodology: This is a cross-sectional study conducted in selected schools in Abha City between December 2016-May 2017. Students from fourth, fifth and sixth classes were included in the study. A structured questionnaire was used to collect the data about knowledge attitude and practices of personal hygiene. Results: The study included 508 primary school children. More than $95 \%$ of the students were aware that the disease can spread from one person to another person. More than $90 \%$ of the students wash their hands with soap before and after eating and also after toilet. Only $71.7 \%$ of students brush their teeth twice daily. There was a positive linear correlation between the knowledge score with practice score and also attitude score with practice score. Conclusion: knowledge attitude and practices concerning personal hygiene among primary school children was satisfactory but still lacking in some aspects.
\end{abstract}

Keywords: Attitude, Hygiene, Knowledge, Practice, Primary School Children

\section{Introduction}

Most of the health problems affecting school students are preventable by promoting proper hygiene practices through family and adopting good health education. Hygiene is defined as the usual practices that are very much interrelated and required for maintaining the health and healthy living (1). The hygienic practices includes taking bath, washing hands, brushing teeth, using soap, brush, cutting nails and caring for gums (2). Hygiene is the discipline which deals about maintaining the health and it is a personal determinant of health which varies according one's moral and practices. Personal hygiene is also influenced by various factors such as society, family and individual 3215 awareness and attitudes concerning hygiene (3). The burden of communicable diseases has been increased because of poor personal hygienic practices and it is considered to be one of the major public health concerns in developing countries (4). School children are more vulnerable because of lack of awareness and as a result of it tremendous increase in the incidence of communicable diseases $(5,6)$. School health is an important intervention which has a major effect on children's health (7) and teachers play a dynamic role in teaching the children about the primordial prevention which chances their attitude and behavior towards personal hygiene. The school becomes the vital place for health promotion activities for children because of its supportive environment and also it is a place where the infectious diseases are likely to spread from one to another $(8,9)$. World health organization report says that 1.7 million deaths occur every year across the world due to lack of personal hygiene (10).

In developing countries acute respiratory infections and intestinal infections are the major reason for the childhood morbidity and mortality whereas diarrheal disease accounts for $12 \%$ of the deaths among under 5 year children in Egypt (11-13). Despite Saudi Arabia being a high income country the prevalence of parasitic infections and intestinal infections are quite high $(14,15)$. The study done in Saudi Arabia by Hamdan Ibrahim Al-Mohammed et al among the primary school children relieved that the prevalence of parasitic infection was $27.2 \%$ among the rural students and the logistic regression analysis found a positive correlation with poor personal hygienic practices (16). Several studies have demonstrated the connection between absence of diseases and good personal hygiene among primary school children and it also reduces the childhood morbidity and mortality due to infectious diseases $(17,18)$. Some other studies also reports that school children with good knowledge and practices are less likely to be absent in schools and more likely to succeed with higher grades $(5,19)$.

Therefore the current study aimed at assessing the knowledge, attitude and practices of the personal hygiene among the male primary school children in Abha, Saudi Arabia. 
Materials \& Methods

Study Design: This is a cross-sectional study.

Study Setting: This study was conducted in the Abha City, Asser Region from December 2016 to May 2017.

Participants: The sample size was calculated by the Epi Info statistical program. The hypothesized frequency of outcome factor in the population is $50 \%$ at a significance level of 0.05 , a design effect of 1 and confidence interval of $95 \%$ ending with a minimum sample size equal 384. Five hundred and eight children were chosen by multistage probability sample technique. The permission was obtained from ministry of education for conducting this research in the primary schools in the Abha city. There are forty primary schools in the Abha city and list of schools with the current location were collected from the ministry of education. First Abha city was divided into east, west, north and south administrations and schools were listed based on these four administrative directions. Two schools from each administrative direction were selected by using a simple random sampling technique. In each school students from class four, five and six were randomly included in the study.

Ethical Permission: Ethical permission was obtained from the research ethics committee, college of medicine, King Khalid University, Abha, Kingdom of Saudi Arabia.

Inclusion Criteria: Primary school children who are studying fourth, fifth and sixth class from the selected schools are included in the study.

Exclusion Criteria: Children who are mentally and physically challenged from the selected schools were excluded.

Tools for Data Collection: Each student was interviewed using a structured questionnaire.

Knowledge Questions: 6 questions about personal hygiene which includes disease spread, boiling water, covering food items, waste disposal, pesticide spray and stagnant water mosquito breeding.

Practice Questions: 16 questions about drinking water, hand washing, covering mouth during sneezing and coughing, taking bath, use soap, sharing food while sick, brush teeth, using tooth brush, wear sandals, cut nails and waste disposal.

Attitude Questions: 3 questions about environmental cleanliness and willingness to share and improve the knowledge about personal hygiene. The questionnaire was framed in English, translated in Arabic (local language) and back translated in English to check the translation.
Reliability: Test-retest reliability was done by Alpha (Cronbach's) test reliability for internal consistency and it was equal to 0.72 . Test reliability was applied on a pilot of 50 primary school children before the study.

Data Analysis: The data analysis was carried out by suing SPSS (Version 17.0, SPSS Inc. Chicago, IL, USA). Frequency tables were used to describe the knowledge, attitude and practices of personal hygiene among the primary school children. Qualitative data were summarized in percentages and non-parametric tests of significance (Chi square test) were applied for nominal scale. Pearson Correlation between two variables was applied. The p-value was two-tailed and statistical significance was set at $<0.05$.

\section{Scoring System for Knowledge, Attitude and Practices: \\ Knowledge}

There are six questions scored $(1 \& 0)$ for yes and no answers and $(1 \& 0)$ for true and false answers. The total score ranged from 0 to 6 . The score was divided into three categories good poor knowledge $(<3)$, average knowledge (3 to 4) and good knowledge (5 to 6 ).

\section{Practice}

There are sixteen questions scored $(1 \& 0)$ for yes and no answers. The total score ranged from 0 to 16 . The score was divided into three categories poor practice $(<9)$, average practice $(9-12)$ and good practice (13-16).

\section{Attitude}

There are three questions, $(1 \& 0)$ for true and false answers, $(1 \& 0)$ for agree and disagree answers and scored $(1 \& 0)$ for yes and no answers. The total score ranged from 0 to 3 . The score was divided into three categories poor attitude (1), average attitude (2) and good attitude (3).

\section{Results}

The study included 508 children studying fourth, fifth and sixth class from eight schools in Abha city. Table 1 shows frequency distribution of the study subjects according to their Knowledge towards personal hygiene; $96.1 \%$ of the students know that the disease spread from one person to another. 23.2 $\%$ of the students were not aware that germs are killed by boiling the water. Around $30 \%$ of students were not having any knowledge about waste disposal. More than $30 \%$ of the students were not known about stagnant water, mosquito breeding and spraying pesticides.

Table 2 shows frequency distribution of the study subjects according to their attitude towards personal hygiene; Around $97 \%$ of students are having a good attitude towards personal hygiene and environmental cleanliness. More than $84 \%$ of 
students are willing to share their knowledge about personal hygiene. Around $90 \%$ of the students are willing to upgrade their knowledge about personal hygiene. Table 3 shows frequency distribution of the study subjects according to their Practice of personal hygiene; only $72 \%$ of the students drink mineral water. On an average $93-97 \%$ of the students wash their hands with soap before and after eating and also after defecating. Only $36 \%$ of the students share their food during illness and only $44.7 \%$ of the students take bath every day. Around $90 \%$ of students use tooth brush whereas only $71.7 \%$ of the students brush their teeth twice daily.

Around $85 \%$ of the students cut their nails regularly and more than $90 \%$ of students dispose their waste through municipal waste collectors. Table 4 shows distribution of students according to knowledge, practice and attitude of personal hygiene; only about $50-56 \%$ of the students have good knowledge and practices about personal hygiene whereas around $75 \%$ of students have good attitude towards personal hygiene.

\section{Correlation between Knowledge Attitude and Practices}

Pearson correlation revealed a significant positive linear correlation between knowledge-practice $(r=$ $0.106, \mathrm{p}=0.017)$, attitude-practice $(\mathrm{r}=0.367, \mathrm{p}$ $<0.001)$. This result confirms the positive relationship between knowledge attitude and practices of personal hygiene as show in the Table 5. The significant association was found between the knowledge score and practices in terms of Drinking tap water $(\mathrm{p}=0.018)$, Drink mineral water $(\mathrm{p}=$ $0.013)$, wash hands after meal $(\mathrm{p}=0.003)$ after toilet $(\mathrm{p}<0.001)$ after sneezing and coughing $(\mathrm{p}<0.001)$, cover mouth during coughing and sneezing ( $\mathrm{p}=$ $0.002)$, share food while sick $(\mathrm{p}=0.001)$, take bath daily $\quad(p=0.001)$, use soap $(p=0.014)$, use tooth brush $(\mathrm{p}=0.010)$ and dispose waste $(\mathrm{p}$ $<0.001$ ) as shown in the Table 6 . Table 7 shows a significant association between the attitude score and knowledge concerning disease spread $(\mathrm{p}=$ $0.049)$ and covering food and water $(\mathrm{p}=0.033)$. There was a significant positive correlation between the attitude score and practices regarding Drink tap water $(\mathrm{p}=0.027)$, wash hands before meal $(\mathrm{p}$ $<0.001)$ after meal $(\mathrm{p}=0.030)$ after toilet $(\mathrm{p}<0.001)$ after coughing and sneezing $(\mathrm{p}<0.001)$ and use soap ( $\mathrm{p}<0.001$ ), cover mouth during coughing and sneezing $(\mathrm{p}=0.015)$, wear sandal $(\mathrm{p}<0.001)$, use tooth brush ( $\mathrm{p}<0.001)$, brush twice daily $(\mathrm{p}<0.001)$ , cut nails $(\mathrm{p}<0.001)$ and dispose waste $(\mathrm{p}<0.001)$. [Table 8]
Table 1: Frequency Distribution of the Study Subjects According to their Knowledge towards Personal Hygiene $(\mathbf{n}=508)$

\begin{tabular}{|c|c|c|}
\hline Questions Asked & $\begin{array}{c}\text { Answer } \\
\text { Level }\end{array}$ & $\begin{array}{c}\text { Total(Percentag } \\
\text { e) }\end{array}$ \\
\hline $\begin{array}{l}\text { Q1. Do you know that } \\
\text { diseases can spread } \\
\text { from one person to } \\
\text { another? }\end{array}$ & $\begin{array}{l}\text { a) Yes } \\
\text { b) } \mathrm{No}\end{array}$ & $\begin{array}{l}\mathrm{N}=488(96.1 \%) \\
\mathrm{N}=20 \quad(3.9 \%)\end{array}$ \\
\hline $\begin{array}{l}\text { Q.2 Does boiling water } \\
\text { kill germs? }\end{array}$ & $\begin{array}{l}\text { a) Yes } \\
\text { b) } \mathrm{No}\end{array}$ & $\begin{array}{l}\mathrm{N}=390(76.8 \%) \\
\mathrm{N}=118(23.2 \%)\end{array}$ \\
\hline $\begin{array}{l}\text { Q.3 Water and food } \\
\text { needs covering }\end{array}$ & $\begin{array}{l}\text { a) Yes } \\
\text { b) } \mathrm{No}\end{array}$ & $\begin{array}{l}\mathrm{N}=449(88.4 \%) \\
\mathrm{N}=59(11.6 \%)\end{array}$ \\
\hline $\begin{array}{l}\text { Q.4 Waste items } \\
\text { should not be thrown } \\
\text { around the house or in } \\
\text { open areas }\end{array}$ & $\begin{array}{c}\text { a) } \\
\text { True } \\
\text { b) } \\
\text { False }\end{array}$ & $\begin{array}{l}\mathrm{N}=354(69.7 \%) \\
\mathrm{N}=154(30.3 \%)\end{array}$ \\
\hline $\begin{array}{l}\text { Q.5 Spraying } \\
\text { pesticides will help } \\
\text { control the spread of } \\
\text { diseases }\end{array}$ & $\begin{array}{c}\text { a) } \\
\text { True } \\
\text { b) } \\
\text { False }\end{array}$ & $\begin{array}{l}\mathrm{N}=282(55.5 \%) \\
\mathrm{N}=226(44.5 \%)\end{array}$ \\
\hline $\begin{array}{l}\text { Q.6 Stagnant water and } \\
\text { dumping areas are } \\
\text { home for mosquitoes }\end{array}$ & $\begin{array}{l}\text { a) } \\
\text { True } \\
\text { b) } \\
\text { False }\end{array}$ & $\begin{array}{l}\mathrm{N}=349(68.7 \%) \\
\mathrm{N}=159(31.3 \%)\end{array}$ \\
\hline
\end{tabular}

Table 2: Frequency Distribution of the Study Subjects According to their Attitude towards Personal Hygiene $(\mathbf{n}=\mathbf{5 0 8})$

\begin{tabular}{|l|l|l|}
\hline \multicolumn{1}{|c|}{ Questions Asked } & \multicolumn{1}{|c|}{$\begin{array}{c}\text { Answer } \\
\text { Level }\end{array}$} & $\begin{array}{c}\text { Total(Percentag } \\
\text { e) }\end{array}$ \\
\hline $\begin{array}{l}\text { Q.23 Personal hygiene } \\
\text { and clean surroundings } \\
\text { are important for } \\
\text { healthy living }\end{array}$ & $\begin{array}{l}\text { a) True } \\
\text { b) False }\end{array}$ & $\begin{array}{l}\mathrm{N}=494(97.2 \%) \\
\mathrm{N}=14(2.8 \%)\end{array}$ \\
\hline $\begin{array}{l}\text { Q.24 Sharing } \\
\text { knowledge about } \\
\text { healthy practices will } \\
\text { lead to a disease free } \\
\text { society? }\end{array}$ & $\begin{array}{l}\text { a) Agree } \\
\text { b) }\end{array}$ & $\begin{array}{l}\mathrm{N}=431(84.8 \%) \\
\mathrm{N}=77(15.2 \%)\end{array}$ \\
\hline $\begin{array}{l}\text { Q.25 I am interested in } \\
\text { increasing my } \\
\text { knowledge about } \\
\text { hygiene measures }\end{array}$ & a) Yes & $\mathrm{N}=458(90.2 \%)$ \\
$\mathrm{n}=50 \quad$ No & & \\
\hline
\end{tabular}


Table 3: Frequency Distribution of the Study Subjects According to their Practice of Personal Hygiene $(\mathbf{n}=\mathbf{5 0 8})$

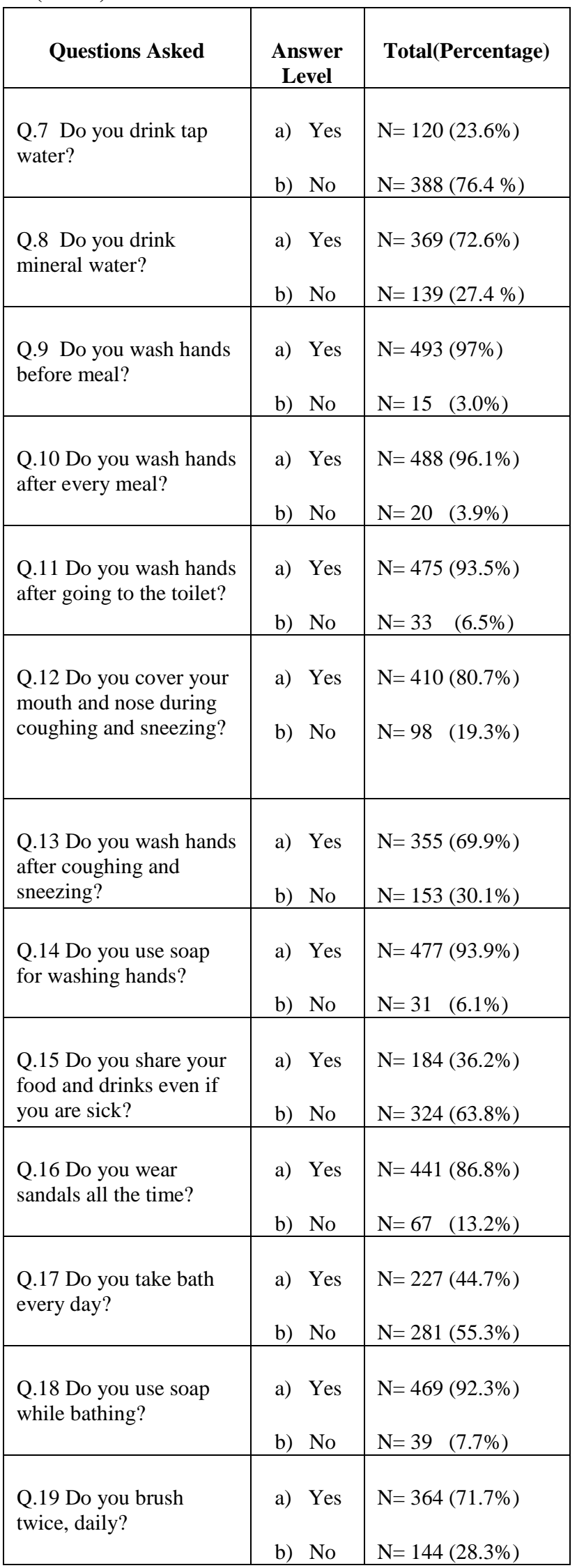

\begin{tabular}{|l|l|l|}
\hline $\begin{array}{l}\text { Q.20 Do you use tooth } \\
\text { brush and paste while } \\
\text { brushing? }\end{array}$ & a) Yes & $\mathrm{N}=454(89.4 \%)$ \\
\hline $\begin{array}{l}\text { Q.21 Do you cut your } \\
\text { nails regularly? }\end{array}$ & a) Yes & $\mathrm{N}=436(85.8 \%)$ \\
\hline $\begin{array}{l}\text { Q.22 Do you dispose } \\
\text { waste to municipal waste } \\
\text { collectors? }\end{array}$ & a) Yes & $\mathrm{N}=464(91.3 \%)$ \\
\hline
\end{tabular}

Table 4: Distribution of Students According to Knowledge, Practice and Attitude of Personal Hygiene $(\mathbf{n}=508)$

\begin{tabular}{|l|l|}
\hline \multicolumn{2}{|c|}{ Hygiene (n= 508) } \\
\hline Knowledge Score & Total (Percentage) \\
\hline Poor (<3) & $22(4.3 \%)$ \\
\hline Average (3-4) & $200(39.4 \%)$ \\
\hline Good (5-6) & $286(56.3 \%)$ \\
\hline Practice Score & \\
\hline Poor (<9) & $24(4.7 \%)$ \\
\hline Average (9-12) & $224(44.1 \%)$ \\
\hline Good (13-16) & $260(51.2 \%)$ \\
\hline Attitude Score & \\
\hline Poor (1) & $13(2.6 \%)$ \\
\hline Average (2) & \\
\hline Good (3) & \\
\hline
\end{tabular}

Table 5: Correlation between Knowledge, Attitude and Practice Scores

\begin{tabular}{|c|c|c|}
\hline Variables & $\begin{array}{c}\text { Correlation } \\
\text { Coefficient }\end{array}$ & p-value* \\
\hline $\begin{array}{c}\text { Knowledge- } \\
\text { Practice }\end{array}$ & 0.106 & $0.017^{*}$ \\
\hline $\begin{array}{c}\text { Knowledge- } \\
\text { Attitude }\end{array}$ & 0.071 & 0.112 \\
\hline Attitude-Practice & 0.367 & $<0.001^{*}$ \\
\hline
\end{tabular}

$* \mathrm{P}<0.05$, Statistically Significant

\section{Discussion}

The current study was conducted in selected primary schools in Abha City and 508 students from fourth, fifth and sixth class were assessed about knowledge, 
attitude and practices of personal hygiene. In the current study more than $95 \%$ of the students were aware about the requirements of personal hygiene and this was slightly higher than the study conducted by Hala $M$ Elsabagh et al in Egypt among the primary school children (20) and the result was almost similar in another study carried out in Erbil city in 2012 in primary school children (21). Only $76 \%$ of the students in this study were known about boiling water kill germs and around $88 \%$ of the students were knowledgeable about covering food and water

Table 6: Correlation Between the Knowledge Score and Practice $(n=508)$

\begin{tabular}{|c|c|c|c|c|c|c|}
\hline \multirow{2}{*}{$\begin{array}{c}\text { Questions } \\
\text { Asked }\end{array}$} & \multirow{2}{*}{$\begin{array}{c}\text { Answer } \\
\text { Levels }\end{array}$} & \multicolumn{3}{|c|}{ Knowledge Score } & \multirow{2}{*}{$\begin{array}{c}\text { Total } \\
\text { (Percent) }\end{array}$} & \multirow{2}{*}{ P Value } \\
\hline & & Poor & Average & Good & & \\
\hline Q.7 & $\begin{array}{l}\text { A. } \\
\text { B. }\end{array}$ & $\begin{array}{l}07(1.38 \%) \\
15(2.95 \%)\end{array}$ & $\begin{array}{c}60(11.81 \%) \\
140(27.56 \%)\end{array}$ & $\begin{array}{c}53(10.43 \%) \\
233(45.87 \%)\end{array}$ & $\begin{array}{l}120(23.62 \%) \\
388(76.38 \%)\end{array}$ & $0.018^{*}$ \\
\hline Q.8 & $\begin{array}{l}\text { A. } \\
\text { B. }\end{array}$ & $\begin{array}{c}9(1.77 \%) \\
13(2.56 \%)\end{array}$ & $\begin{array}{c}148(29.13 \%) \\
52(10.24 \%)\end{array}$ & $\begin{array}{c}212(41.73 \%) \\
74(14.57 \%)\end{array}$ & $\begin{array}{l}369(72.63 \%) \\
139(27.37 \%)\end{array}$ & $0.013^{*}$ \\
\hline Q.9 & $\begin{array}{l}\text { A. } \\
\text { B. }\end{array}$ & $\begin{array}{c}22(4.33 \%) \\
0(0.00 \%)\end{array}$ & $\begin{array}{c}194(38.19 \%) \\
06(1.18 \%)\end{array}$ & $\begin{array}{c}277(54.53 \%) \\
09(1.77 \%)\end{array}$ & $\begin{array}{c}493(97.05 \%) \\
15(2.95 \%)\end{array}$ & 0.869 \\
\hline Q.10 & $\begin{array}{l}\text { A. } \\
\text { B. }\end{array}$ & $\begin{array}{l}20(3.94 \%) \\
02(0.39 \%)\end{array}$ & $\begin{array}{c}188(37.01 \%) \\
12(2.36 \%)\end{array}$ & $\begin{array}{c}280(55.12 \%) \\
06(1.18 \%)\end{array}$ & $\begin{array}{c}488(96.07 \%) \\
20(3.93 \%)\end{array}$ & $0.003^{*}$ \\
\hline Q.11 & $\begin{array}{l}\text { A. } \\
\text { B. }\end{array}$ & $\begin{array}{l}20(3.94 \%) \\
02(0.39 \%)\end{array}$ & $\begin{array}{c}179(35.24 \%) \\
21(4.13 \%)\end{array}$ & $\begin{array}{c}276(54.33 \%) \\
10(1.97 \%)\end{array}$ & $\begin{array}{c}475(93.51 \%) \\
33(6.49 \%)\end{array}$ & $<0.001 *$ \\
\hline Q.12 & $\begin{array}{l}\text { A. } \\
\text { B. }\end{array}$ & $\begin{array}{l}12(2.36 \%) \\
10(1.97 \%)\end{array}$ & $\begin{array}{c}153(30.12 \%) \\
47(9.25 \%)\end{array}$ & $\begin{array}{c}245(48.23 \%) \\
41(8.07 \%)\end{array}$ & $\begin{array}{c}410(80.71 \%) \\
98(19.29 \%)\end{array}$ & $0.002 *$ \\
\hline Q.13 & $\begin{array}{l}\text { A. } \\
\text { B. }\end{array}$ & $\begin{array}{l}13(2.56 \%) \\
09(1.77 \%)\end{array}$ & $\begin{array}{c}131(25.79 \%) \\
69(13.58 \%)\end{array}$ & $\begin{array}{l}211(41.54 \%) \\
75(14.76 \%)\end{array}$ & $\begin{array}{l}355(69.88 \%) \\
153(30.12 \%)\end{array}$ & $<0.001^{*}$ \\
\hline Q.14 & $\begin{array}{l}\text { A. } \\
\text { B. }\end{array}$ & $\begin{array}{c}22(4.33 \%) \\
0(0.00 \%)\end{array}$ & $\begin{array}{c}190(37.40 \%) \\
10(1.97 \%)\end{array}$ & $\begin{array}{c}265(52.17 \%) \\
21(4.13 \%)\end{array}$ & $\begin{array}{c}477(93.90 \%) \\
31(6.10 \%)\end{array}$ & 0.090 \\
\hline Q.15 & $\begin{array}{l}\text { A. } \\
\text { B. }\end{array}$ & $\begin{array}{l}17(3.35 \%) \\
05(0.98 \%)\end{array}$ & $\begin{array}{c}72(14.17 \%) \\
128(25.20 \%)\end{array}$ & $\begin{array}{l}95(18.70 \%) \\
191(37.60 \%)\end{array}$ & $\begin{array}{l}184(36.22 \%) \\
324(63.78 \%)\end{array}$ & $0.001 *$ \\
\hline Q.16 & $\begin{array}{l}\text { A. } \\
\text { B. }\end{array}$ & $\begin{array}{l}18(3.54 \%) \\
04(0.79 \%)\end{array}$ & $\begin{array}{c}175(34.45 \%) \\
25(4.92 \%)\end{array}$ & $\begin{array}{c}248(48.82 \%) \\
38(7.48 \%)\end{array}$ & $\begin{array}{c}441(86.81 \%) \\
67(13.19 \%)\end{array}$ & 0.187 \\
\hline Q.17 & $\begin{array}{l}\text { A. } \\
\text { B. }\end{array}$ & $\begin{array}{l}04(0.79 \%) \\
18(3.54 \%)\end{array}$ & $\begin{array}{c}94(18.50 \%) \\
106(20.87 \%)\end{array}$ & $\begin{array}{l}129(25.39 \%) \\
157(30.91 \%)\end{array}$ & $\begin{array}{l}227(44.69 \%) \\
281(55.31 \%)\end{array}$ & $0.001^{*}$ \\
\hline Q.18 & $\begin{array}{l}\text { A. } \\
\text { B. }\end{array}$ & $\begin{array}{l}20(3.94 \%) \\
02(0.39 \%)\end{array}$ & $\begin{array}{c}184(36.22 \%) \\
16(3.15 \%)\end{array}$ & $\begin{array}{c}265(52.17 \%) \\
21(4.13 \%)\end{array}$ & $\begin{array}{c}469(92.32 \%) \\
39(7.68 \%)\end{array}$ & $0.014^{*}$ \\
\hline Q.19 & $\begin{array}{l}\text { A. } \\
\text { B. }\end{array}$ & $\begin{array}{l}13(2.56 \%) \\
09(1.77 \%)\end{array}$ & $\begin{array}{c}139(27.36 \%) \\
61(12.01 \%)\end{array}$ & $\begin{array}{c}212(41.73 \%) \\
74(14.57 \%)\end{array}$ & $\begin{array}{l}364(71.65 \%) \\
144(28.35 \%)\end{array}$ & 0.596 \\
\hline Q.20 & $\begin{array}{l}\text { A. } \\
\text { B. }\end{array}$ & $\begin{array}{l}17(3.35 \%) \\
05(0.98 \%)\end{array}$ & $\begin{array}{c}171(33.66 \%) \\
29(5.71 \%)\end{array}$ & $\begin{array}{c}266(52.36 \%) \\
20(3.94 \%)\end{array}$ & $\begin{array}{c}454(89.37 \%) \\
54(10.63 \%)\end{array}$ & $0.010^{*}$ \\
\hline Q.21 & $\begin{array}{l}\text { A. } \\
\text { B. }\end{array}$ & $\begin{array}{l}18(3.54 \%) \\
04(0.79 \%)\end{array}$ & $\begin{array}{c}177(34.84 \%) \\
23(4.53 \%)\end{array}$ & $\begin{array}{c}241(47.44 \%) \\
45(8.86 \%)\end{array}$ & $\begin{array}{c}436(85.83 \%) \\
72(14.17 \%)\end{array}$ & 0.243 \\
\hline Q.22 & $\begin{array}{l}\text { A. } \\
\text { B. }\end{array}$ & $\begin{array}{l}14(2.76 \%) \\
08(1.57 \%)\end{array}$ & $\begin{array}{c}181(52.95 \%) \\
19(3.35 \%)\end{array}$ & $\begin{array}{c}269(52.95 \%) \\
17(3.35 \%)\end{array}$ & $\begin{array}{c}464(91.34 \%) \\
44(8.66 \%)\end{array}$ & $<0.001^{*}$ \\
\hline
\end{tabular}

$* \mathrm{P}<0.05$, Statistically Significant 
Helix Vol. 8(2): 3215-3223

Table 7: Correlation Between the Attitude Score and Practice $(\mathbf{n}=508)$

\begin{tabular}{|c|c|c|c|c|c|c|}
\hline \multirow{2}{*}{$\begin{array}{c}\text { Questions } \\
\text { Asked }\end{array}$} & \multirow{2}{*}{$\begin{array}{c}\text { Answer } \\
\text { Levels }\end{array}$} & \multicolumn{3}{|c|}{ Knowledge Score } & \multirow{2}{*}{$\begin{array}{c}\text { Total } \\
\text { (Percent) }\end{array}$} & \multirow{2}{*}{ P Value } \\
\hline & & Poor & Average & Good & & \\
\hline Q.7 & $\begin{array}{l}\text { A. } \\
\text { B. }\end{array}$ & $\begin{array}{l}07(1.38 \%) \\
06(1.18 \%)\end{array}$ & $\begin{array}{c}29(5.71 \%) \\
86(16.93 \%)\end{array}$ & $\begin{array}{c}84(16.54 \%) \\
296(58.27 \%)\end{array}$ & $\begin{array}{l}120(23.62 \%) \\
388(76.38 \%)\end{array}$ & $0.027^{*}$ \\
\hline Q.8 & $\begin{array}{l}\text { A. } \\
\text { B. }\end{array}$ & $\begin{array}{l}1(2.17 \%) \\
02(0.39 \%)\end{array}$ & $\begin{array}{c}81(15.94 \%) \\
34(6.69 \%)\end{array}$ & $\begin{array}{l}277(54.53 \%) \\
103(20.28 \%)\end{array}$ & $\begin{array}{l}369(72.64 \%) \\
139(27.36 \%)\end{array}$ & 0.540 \\
\hline Q.9 & $\begin{array}{l}\text { A. } \\
\text { B. }\end{array}$ & $\begin{array}{l}07(1.38 \%) \\
06(1.18 \%)\end{array}$ & $\begin{array}{c}108(21.26 \%) \\
07(1.38 \%)\end{array}$ & $\begin{array}{c}378(74.41 \%) \\
02(0.39 \%)\end{array}$ & $\begin{array}{c}493(97.05 \%) \\
15(2.95 \%)\end{array}$ & $<0.001^{*}$ \\
\hline Q.10 & $\begin{array}{l}\text { A. } \\
\text { B. }\end{array}$ & $\begin{array}{l}11(2.17 \%) \\
02(0.39 \%)\end{array}$ & $\begin{array}{c}108(21.26 \%) \\
07(1.38 \%)\end{array}$ & $\begin{array}{c}369(72.64 \%) \\
11(2.17 \%)\end{array}$ & $\begin{array}{c}488(96.06 \%) \\
20(3.94 \%)\end{array}$ & $0.030^{*}$ \\
\hline Q.11 & $\begin{array}{l}\text { A. } \\
\text { B. }\end{array}$ & $\begin{array}{l}08(1.57 \%) \\
05(0.98 \%)\end{array}$ & $\begin{array}{c}106(20.87 \%) \\
09(1.77 \%)\end{array}$ & $\begin{array}{c}361(71.06 \%) \\
19(3.74 \%)\end{array}$ & $\begin{array}{c}475(93.50 \%) \\
33(6.50 \%)\end{array}$ & $<0.001^{*}$ \\
\hline Q.12 & $\begin{array}{l}\text { A. } \\
\text { B. }\end{array}$ & $\begin{array}{l}1(2.17 \%) \\
02(0.39 \%)\end{array}$ & $\begin{array}{c}82(16.14 \%) \\
33(6.50 \%)\end{array}$ & $\begin{array}{c}317(62.40 \%) \\
63(12.40 \%)\end{array}$ & $\begin{array}{c}410(80.71 \%) \\
98(19.29 \%)\end{array}$ & $0.015^{*}$ \\
\hline Q.13 & $\begin{array}{l}\text { A. } \\
\text { B. }\end{array}$ & $\begin{array}{l}04(0.79 \%) \\
09(1.77 \%)\end{array}$ & $\begin{array}{l}71(13.98 \%) \\
44(8.66 \%)\end{array}$ & $\begin{array}{l}280(55.12 \%) \\
100(19.69 \%)\end{array}$ & $\begin{array}{l}355(69.88 \%) \\
153(30.12 \%)\end{array}$ & $<0.001^{*}$ \\
\hline Q.14 & $\begin{array}{l}\text { A. } \\
\text { B. }\end{array}$ & $\begin{array}{l}09(1.77 \%) \\
04(0.79 \%)\end{array}$ & $\begin{array}{c}96(18.90 \%) \\
19(3.74 \%)\end{array}$ & $\begin{array}{c}372(73.23 \%) \\
08(1.57 \%)\end{array}$ & $\begin{array}{c}477(93.90 \%) \\
31(6.10 \%)\end{array}$ & $<0.001^{*}$ \\
\hline Q.15 & $\begin{array}{l}\text { A. } \\
\text { B. }\end{array}$ & $\begin{array}{l}06(1.18 \%) \\
07(1.38 \%)\end{array}$ & $\begin{array}{c}44(8.66 \%) \\
71(13.98 \%)\end{array}$ & $\begin{array}{l}134(26.38 \%) \\
246(48.43 \%)\end{array}$ & $\begin{array}{l}184(36.22 \%) \\
324(63.78 \%)\end{array}$ & 0.633 \\
\hline Q.16 & $\begin{array}{l}\text { A. } \\
\text { B. }\end{array}$ & $\begin{array}{l}08(1.57 \%) \\
05(0.98 \%)\end{array}$ & $\begin{array}{c}89(17.52 \%) \\
26(5.12 \%)\end{array}$ & $\begin{array}{c}344(67.72 \%) \\
36(7.09 \%)\end{array}$ & $\begin{array}{c}441(86.81 \%) \\
67(13.19 \%)\end{array}$ & $<0.001^{*}$ \\
\hline Q.17 & $\begin{array}{l}\text { A. } \\
\text { B. }\end{array}$ & $\begin{array}{l}06(1.18 \%) \\
07(1.38 \%)\end{array}$ & $\begin{array}{c}44(8.66 \%) \\
71(13.98 \%)\end{array}$ & $\begin{array}{l}177(34.84 \%) \\
203(39.96 \%)\end{array}$ & $\begin{array}{l}227(44.69 \%) \\
281(55.31 \%)\end{array}$ & 0.289 \\
\hline Q.18 & $\begin{array}{l}\text { A. } \\
\text { B. }\end{array}$ & $\begin{array}{l}1(2.17 \%) \\
02(0.39 \%)\end{array}$ & $\begin{array}{c}101(19.88 \%) \\
14(2.76 \%)\end{array}$ & $\begin{array}{c}357(70.28 \%) \\
23(4.53 \%)\end{array}$ & $\begin{array}{c}469(92.32 \%) \\
39(7.68 \%)\end{array}$ & 0.055 \\
\hline Q.19 & $\begin{array}{l}\text { A. } \\
\text { B. }\end{array}$ & $\begin{array}{l}04(0.79 \%) \\
09(1.77 \%)\end{array}$ & $\begin{array}{l}59(11.61 \%) \\
56(11.02 \%)\end{array}$ & $\begin{array}{c}301(59.25 \%) \\
79(15.55 \%)\end{array}$ & $\begin{array}{l}364(71.65 \%) \\
144(28.35 \%)\end{array}$ & $<0.001^{*}$ \\
\hline Q.20 & $\begin{array}{l}\text { A. } \\
\text { B. }\end{array}$ & $\begin{array}{l}07(1.38 \%) \\
06(1.18 \%)\end{array}$ & $\begin{array}{c}98(19.29 \%) \\
17(3.35 \%)\end{array}$ & $\begin{array}{c}349(68.70 \%) \\
31(6.10 \%)\end{array}$ & $\begin{array}{c}454(89.37 \%) \\
54(10.63 \%)\end{array}$ & $<0.001^{*}$ \\
\hline Q.21 & $\begin{array}{l}\text { A. } \\
\text { B. }\end{array}$ & $\begin{array}{l}06(1.18 \%) \\
07(1.38 \%)\end{array}$ & $\begin{array}{c}85(16.73 \%) \\
30(5.91 \%)\end{array}$ & $\begin{array}{c}345(67.91 \%) \\
35(6.89 \%)\end{array}$ & $\begin{array}{c}436(85.83 \%) \\
72(14.17 \%)\end{array}$ & $<0.001^{*}$ \\
\hline Q.22 & $\begin{array}{l}\text { A. } \\
\text { B. }\end{array}$ & $\begin{array}{l}05(0.98 \%) \\
08(1.57 \%)\end{array}$ & $\begin{array}{c}99(19.49 \%) \\
16(3.15 \%)\end{array}$ & $\begin{array}{c}360(70.87 \%) \\
20(3.94 \%)\end{array}$ & $\begin{array}{c}464(91.34 \%) \\
44(8.66 \%)\end{array}$ & $<0.001 *$ \\
\hline
\end{tabular}

$* \mathrm{P}<0.05$, Statistically Significant 
Table 8: Correlation Between the Attitude Score and Knowledge $(n=508)$

\begin{tabular}{|c|c|c|c|c|c|c|}
\hline \multirow{2}{*}{$\begin{array}{c}\text { Questions } \\
\text { Asked }\end{array}$} & \multirow{2}{*}{$\begin{array}{c}\text { Answer } \\
\text { Levels }\end{array}$} & \multicolumn{3}{|c|}{ Knowledge Score } & \multirow{2}{*}{$\begin{array}{c}\text { Total } \\
\text { (Percent) }\end{array}$} & \multirow{2}{*}{ P Value } \\
\hline & & Poor & Average & Good & & \\
\hline Q.1 & $\begin{array}{l}\text { A. } \\
\text { B. }\end{array}$ & $\begin{array}{l}11(2.17 \%) \\
02(0.39 \%)\end{array}$ & $\begin{array}{c}113(22.24 \%) \\
02(0.39 \%)\end{array}$ & $\begin{array}{c}364(71.65 \%) \\
16(3.15 \%)\end{array}$ & $\begin{array}{c}488(96.06 \%) \\
20(3.94 \%)\end{array}$ & $0.049 *$ \\
\hline Q.2 & $\begin{array}{l}\text { A. } \\
\text { B. }\end{array}$ & $\begin{array}{l}07(1.38 \%) \\
06(1.18 \%)\end{array}$ & $\begin{array}{c}94(18.50 \%) \\
21(4.13 \%)\end{array}$ & $\begin{array}{c}289(56.89 \%) \\
91(17.91 \%)\end{array}$ & $\begin{array}{l}390(76.77 \%) \\
118(23.23 \%)\end{array}$ & 0.063 \\
\hline Q.3 & $\begin{array}{l}\text { A. } \\
\text { B. }\end{array}$ & $\begin{array}{l}11(2.17 \%) \\
02(0.39 \%)\end{array}$ & $\begin{array}{c}94(18.50 \%) \\
21(4.13 \%) \\
\end{array}$ & $\begin{array}{c}344(67.72 \%) \\
36(7.09 \%)\end{array}$ & $\begin{array}{c}449(88.39 \%) \\
59(11.61 \%)\end{array}$ & $0.033 *$ \\
\hline Q.4 & $\begin{array}{l}\text { A. } \\
\text { B. }\end{array}$ & $\begin{array}{l}10(1.97 \%) \\
03(0.59 \%)\end{array}$ & $\begin{array}{c}78(15.35 \%) \\
37(7.28 \%)\end{array}$ & $\begin{array}{c}266(52.36 \%) \\
114(22.44)\end{array}$ & $\begin{array}{l}354(69.69 \%) \\
154(30.31 \%)\end{array}$ & 0.768 \\
\hline Q.5 & $\begin{array}{l}\text { A. } \\
\text { B. }\end{array}$ & $\begin{array}{l}08(1.57 \%) \\
05(0.98 \%)\end{array}$ & $\begin{array}{l}57(11.22 \%) \\
58(11.42 \%) \\
\end{array}$ & $\begin{array}{l}217(42.72 \%) \\
163(32.09 \%)\end{array}$ & $\begin{array}{l}282(55.51 \%) \\
226(44.49 \%)\end{array}$ & 0.328 \\
\hline Q.6 & $\begin{array}{l}\text { A. } \\
\text { B. }\end{array}$ & $\begin{array}{l}07(1.38 \%) \\
06(7.48 \%)\end{array}$ & $\begin{array}{c}77(15.16 \%) \\
38(7.48 \%)\end{array}$ & $\begin{array}{l}265(52.17 \%) \\
115(22.64 \%)\end{array}$ & $\begin{array}{l}349(68.70 \%) \\
159(31.30 \%)\end{array}$ & 0.430 \\
\hline
\end{tabular}

$* \mathrm{P}<0.05$, Statistically Significant

These results were slightly higher than the study conducted by Vivas et al in 2010 among the Ethiopian primary school children (5). Hand washing practices among the students in the current study is extraordinary; more than $90 \%$ of the students wash their hands before and after meals and also after defecating and this is higher than the study aimed at assessing the factors influencing the hygiene behavior among school children in Ethiopia by Assefa $M$ et al in 2014 (22). In terms of hand washing practices our study results were higher than other studies conducted in Ghana, Colombia and Kenya (23-25). The current study reports more than $90 \%$ of the children use soap for hand washing and bathing and this was in agreement with previous published studies $(21,26)$. However use of soap by the primary school students for washing hands and bathing was higher than the study conducted in India $(85.4 \%)$ and another one in turkey $(46.9 \%)$ respectively $(27,28)$. This study shows only $44 \%$ of the primary school children take bath daily which is lesser than the other reported studies in India ${ }^{20}$ and Egypt (26). In this study around $90 \%$ of the students used tooth brush and paste whereas only $71 \%$ of them brush their teeth twice daily. A study conducted by Kenneth et al in 2008 among the school children found that more than $75 \%$ of the children in Switzerland, Sweden, Netherland, Germany, Denmark and Norway brushed their teeth twice daily. However this was higher than our study results (29).

Another study by Peterson et al revealed that less than $46 \%$ of children in Finland, Romania, Greece, Lithuania, Turkey and Malta brush their teeth more than once (30). About $85 \%$ of the students cut their nails regularly which were very much higher when compared to a study conducted in India which showed only $30 \%$ of the students routinely cut their nails (31). This study found that only $50-56 \%$ of the students had good knowledge and good practices of personal hygiene which was higher than the study conducted in Egypt by Hala M Elsabagh et al (20). Around $75 \%$ of the students were with good attitude towards personal hygiene which was in concurrence with the study carried out in United Arab Emirates (32). The correlation revealed significant positive linear correlations between knowledge-practice $(\mathrm{r}=$ $0.106, \mathrm{p}=0.017)$, attitude-practice $(\mathrm{r}=0.367, \mathrm{p}$ $<0.001)$. The positive linear correlation confirms that better knowledge and good attitude can lead to good practices. This will help in prevention of communicable diseases. This study finding are in agreement with another study conducted by Smith et al which reported students with strong knowledge showed better attitude and practices (33). This main finding of the study was also supported by other study done in India by Pragya Pandey Wahengbam et al which also found a positive linear correlation between knowledge attitude and practices (34).

\section{Conclusion}

Primary school children knowledge attitude and practices towards personal hygiene was satisfactory but still deficient in some aspects. The study result shows a positive linear correlation between knowledge attitude and practices so there is a need for health education regarding personal hygiene for the school students for better awareness and developing a good attitude and behavior. Though this type of research has a limitation such as selfreported data and information bias; the findings of this study encourages addition research for creating awareness concerning personal hygiene. The study also provides a baseline data for future research.

\section{References}

1. Khinda PK, Mahajan R, Gill AS, Uppal RS, et al. Assessment of preventive dental care among dental students and dental professionals in India: A knowledge, attitude, and practice study. Saint Int Dent J 2015;1:10511.

2. International Scientific Forum on home hygiene. 2011 (Accessed November 21, 2017) Available at http://www.ifh-homehygiene.org.

3. Postma L, Getkate I, VanwijkC: Life Skill Based Hygiene Education; International water and 
Sanitation Centre, 2004. (Accessed November 21, 2017)Available http://www.ircwash.org/sites/default/files/Postma2004.

4. World Health Organization. Better Health for Poor Children. (Accessed November 21, 2017) Available

at:http://www.who.int/child_adolescent_health/doc uments/a91061/en/index.html.

5. Vivas A, Gelaye B, Aboset N, Kumie A, Berhane Y, Williams MA. Knowledge, Attitudes, and Practices (KAP) of Hygiene among School Children in Angolela, Ethiopia. J Prev Med Hyg. 2010; 51(2): 73-79.

6. Sarkar M. Personal hygiene among primary school children living in a slum of Kolkata, India. Journal of Preventive Medicine and Hygiene. 2013; 54(3):153-158.

7. Ali AE, Fahmy HH, Mohamed SE, El Hawy LL. Effect of health education about healthy diet, physical activity and personal hygiene among governmental primary school children (11-13 years old) in Sharkia Governorate-Egypt. Journal of American Science. 2016;12(6).

8. Deb S, Dutta S, Dasgupta A, Misra R. Relationship of Personal Hygiene with Nutrition and Morbidity Profile: A Study among Primary School Children in South Kolkata. Indian Journal of Community Medicine. 2010; 35(2): 280-284.

9. Varu R B. School Health Services in India. The social and economic context. Sage Publications Pvt. Ltd; 2008.

10. World Health Organization: The world health report 2002 - Reducing Risks, Promoting Healthy Life, 2002 (Accessed November 21, 2017) Available at http://www.who.int/whr/2002/en/.

11. Vivace A., Gelaya B., Aboset N., Kumiec A., et al. Knowledge, Attitudes, and Practices (KAP) of Hygiene among School Children in Angolela, Ethiopia. J Prev Med Hyg. 2010 June; 51(2): 73-79.

12. Egypt census 2014 available at http://www.indexmundi.com/egypt/demographics_ profil e.html (Accessed November 21, 2017).

13. CHERG/WHO/UNICEF for distribution of causes of neonatal and under-five deaths (published in Liu et al, Lancet 2012). (Accessed November 21, 2017)

http://www.who.int/maternal_child_adolescent/epi demio logy/profiles/neonatal_child/egy.pdf
14. Barnawi AB, Tonkal AM, Fouad MA, AlBraiken FA. Detection of Entamoeba histolytica/dispar in stool specimens by using enzyme-linked immunosorbent assay in the population of Jeddah City, Saudi Arabia. J Egypt Soc Parasitol 2007; 37(1): 143-50.

15. El-Sheikh SM, El Assouli SM. Prevalence of viral, bacterial and parasitic enteropathogens among young children with acute diarrhea in Jeddah, Saudi Arabia. J Health Popul Nut 2001; 19(1):25-30.

16. Hamdan Ibrahim Al-Mohammed et al. Prevalence of intestinal parasitic infections and its relationship with socio-demographics and hygienic habits among male primary schoolchildren in AlAhsa, Saudi Arabia. Asian Pacific Journal of Tropical Medicine (2010)906-912.

17. Ahmadu BU, Rimamchika M, Ibrahim A, Nnanubumom AA, Godiya A, Emmanuel P. State of personal hygiene among primary school children: A community based cohort study. Sudanese journal of paediatrics. 2013;13(1):38.

18. Khatoon R, Sachan B, Khan MA, Srivastava JP. Impact of school health education program on personal hygiene among school children of Lucknow district. Journal of family medicine and primary care. 2017 Jan;6(1):97.

19. Water and Sanitation Program Can hygiene be cool and fun: Insights from School Children in Senegal. Available at: http://www.comminit.com/en/node/264152/38.

(Accessed November 21, 2017).

20. Hala M Elsabagh et al. KAP Regarding Personal Hygiene among Preschool Children. Int J Med Res Prof.2016; 2(2); 255-61.

21. Sheren N., AzizK and Abdulla S: Knowledge and attitudes of pupils in some of the primary schools regarding personal hygiene in Erbil city. KUFA JOURNAL FOR NURSING SCIENCES 2012 Vol. 2, Issue1, 5.

22. Assefa M., Kumie A. Assessment of factors influencing hygiene behavior among school children in Mereb-Leke District, Northern Ethiopia: a crosssectional study. BMC Public Health2014, 14:1000.

23. Scott B, Curtis C, Rabie T, Garbrah-Aidoo N. Health in our hands but not in our heads: understanding hygiene motivation in Ghana. Health Policy and Planning. 2007;22:225-233.

24. Lopez-Quintero C, Freeman P, Neumark Y. Hand Washing Among School Children in Bogotá, Colombia. Am J Public Health. 2009; 99: 94-101. 
25. O'Reilly et al. The impact of a school-based safe water and hygiene programme on knowledge and practices of students and their parents: Nyanza Province, western Kenya, 2006.Epidemiol. Infect. $2008 ; 136: 80-91$.

26. Ansari S.Y. Warbhe P.A: Assessment of the Knowledge and Practice Regarding Personal Hygiene among School Children from an Urban Area. International Journal of Current Medical and Applied Sciences, vol.4. Issue 1, September: 2014. PP: 1-12.

27. Shrestha A., Angolkar M. Impact of Health Education on the Knowledge and Practice Regarding Personal Hygiene among Primary School Children in Urban Area of Karnataka, India. IOSR Journal of Dental and Medical Sciences (IOSRJDMS). Volume 13, Issue 4 Ver. VII. (Apr. 2014), PP 86-89.

28. Yalcin SS, Yalcin S, Altin S. Hand washing, and adolescents. A study from seven schools in Konya, Turkey. Int J Adolesc Med Health. 2004; 16:371-6.

29. Eaton K., Carlile M. : Tooth brushing behavior in Europe: opportunities for dental public health. International Dental Journal. ; 2008; 58, 287-293.

30. Nematian J, Nematian E, Gholamrezanezhad A, AsgariAA: Prevalence of intestinal parasitic infections and their relation with socio-economic factors and hygienic habits in Tehran primary school students. Acta Trop.: 2004; 92:179-86.

31. Dongre AR, Deshmukh PR, Garg BS. The impact of school health education programme on personal hygiene and related morbidities in tribal school children of Wardha District. Indian $\mathrm{J}$ Community Med 2006;31:81-2.

32. Dakhili S, Alsuwaidi N, Sara Saeed S, et al. Oral hygiene: association between knowledge and practice among school going children in Ajman, United Arab Emirates. American Journal of Research Communication, 2014, 2(10): 39-48.

33. Smyth E., Caamaño F., Fernández-Riveiro P: Oral health knowledge, attitudes and practice in 12year-old schoolchildren. Med Oral Patol Oral Cir Bucal2007. Dec 1;12(8):E614-20.

34. Pragya Pandey Wahengbam et al., Oral Health in Manipuri Youths. Journal of Clinical and Diagnostic Research. 2016 Jun, Vol-10(6): ZC65ZC70. 